\title{
Numerical experiments in screening theory
}

\author{
G. Shaviv ${ }^{1,2, \star}$ \\ 1 Institut für Theoretische Astrophysik Tiergartenstrasse 15, 69121 Heidelberg, Germany \\ 2 On leave from Department of Physics and Asher Space Research Institute, Israel Institute of Technology Haifa, 32000, Israel
}

Received 15 October 2003 / Accepted 1 February 2004

\begin{abstract}
In an attempt to clarify the behavior of the screening phenomenon in $N$-body systems at thermodynamics equilibrium, under the particular conditions at the core of the Sun, we define the screening energy and summarize the two main types of methods to calculate it: (a) by using classical mean field in Statistical Mechanics and (b) ab initio, by using molecular dynamics and first principles only. We invent a pair interaction with a finite parametrized range and strength, and carry out a numerical experiment which demonstrates the difference between the two aforementioned methods and underlying assumptions as a function of the range of the interaction (or equivalently the number of particles in the interaction zone). We study the effect that fluctuations in the number of particles in the screening/neutralizing cloud have on the screening, and the deviations from mean field results which ensue. We also demonstrate that the classical mean field theory is inaccurate under the conditions prevailing in stellar cores and essentially over estimates the screening. We show how the molecular dynamics results tend to the mean field Statistical Mechanics limits when the number of particles in the neutralizing domain increases.
\end{abstract}

Key words. methods: $N$-body simulations - nuclear reactions, nucleosynthesis, abundances - plasmas - Sun: interior

\section{Introduction}

The rates of the nuclear reactions which take place in dense stellar cores are affected by the environment. The effect is known as the screening factor. The original idea that the environment affects the rate of the reaction is due to Schatzman (1948) and the general theory was worked out by Salpeter (1954). Salpeter (1954) assumed a mean field theory for the screening cloud, the Debye theory, and stated the approximations involved. Salpeter defined the screening as the extra energy gained by a pair of approaching particles over their relative energy at large separation.

Alastuey \& Jancovici (1978) formulated the screening as the enhancement of the population of particles at vanishing separation due to the particles in the screening cloud. Namely, if $g(r)$ is the pair correlation function, then the screening is given by the revised value of $g(0)$ (relative to a pure random distribution) due to the screening cloud in the system. Hence the enhancement is $E=g(0) / g_{0}(0)$, where $g_{0}(r)$ is the pair correlation in infinite dilution.

Any mean field theory tacitly assumes that $N_{\mathrm{D}}=n R_{\mathrm{D}}^{3} \gg 1$, where $N_{\mathrm{D}}$ is the number of particles in the screening cloud (or the Debye sphere in case of a plasma), $n$ is the number density and $R_{\mathrm{D}}$ the Debye radius. The conditions in stars along the main sequence in general, and in the Sun in particular, are such that $N_{\mathrm{D}} \approx 1-3$ and the basic condition for the validity of mean field of the screening cloud is not satisfied (cf. Shaviv \& Shaviv 2002, hereafter SS02). We remark that already Salpeter (1954)

\footnotetext{
^ e-mail: gioras@physics.technion.ac.il
}

pointed out that the mean field assumption which presupposes a sufficiently large number of particles in the effective interaction domain, is in question.

The mean field theory, where each particle possess an effective kinetic energy independent potential, releases us from the need to consider questions such as:

- Each particle in the system is inside a potential well created by the surrounding particles. What happens to the potential wells of the two particles when they approach each other?

- Are the particles which form the potential well of a given particle affected by the incoming fast particle?

- Are the effects independent of the relative kinetic energy?

as well as many others. To what extent are these questions relevant to the screening problem in stars?

Recently Bahcall et al. (2002) (hereafter BBGS) have discussed the Salpeter formula for calculating the electrostatic screening of nuclear reactions in plasma and claim to have shown that it should apply to the Sun and that all claims concerning dynamic effects are wrong, including the recent results of Shaviv \& Shaviv (2001) (hereafter SS01). The discussion of BBGS is based on the assumption that the mean field electrostatic potential is a good assumption ignoring the fact that in the core of the Sun this very assumption may not be justified. Further, the authors assume that two interacting ions always gain energy from the plasma, as the particles approach each other. This additional energy, the screening energy, is taken to be just the mean field potential of the ions in the plasma, namely, the long time average (the thermodynamic 
time - much longer than a typical collision) of the potential felt by an ion in the plasma. As the particles move apart, the tacit assumption is that this extra energy is exactly returned to the plasma.

Shaviv \& Shaviv (SS01, SS02) have shown how to obtain the screening energy from first principles using ab initio Molecular Dynamics (hereafter MD) without assuming the fundamental assumption of the existence of a mean field. SS01 carried out extensive MD simulations that show that on the $a v$ erage particles with low relative energy gain energy from the particles around when they scatter one off the other, while particles with high relative energy lose on the average energy to the particles around as they scatter. The sum of the exchange over all particles vanishes in equilibrium. SS01 showed the connection between the screening and the dynamic friction in the system. However, in view of the on going controversy on the screening problem, which is important to stellar evolution in all phases of nuclear burning, it is imperative to better understand the underlying physics and the effect of the various assumptions.

The basic advantage of Molecular Dynamic is that it is not bound by the validity of the mean field. Hence, it allows the investigation of the fundamental processes in the screening phenomenon. This was done by SS01 \& SS02. Yet, when treating a plasma composed of positive and negative charges and Coulomb forces, the neutralization is determined by the physical conditions. Thus it is difficult to assess the particular effect a certain neutralization radius has. In an attempt to demonstrate the applicability range of the statistical mean field theory as applied to screening and manifest the need for the dynamic approach of Molecular Dynamics, we invent a finite range potential in which the range and the force strength are free parameters. By calculating the screening for different values of the range we can asses the effect of the number of particle in the screening cloud, namely the fluctuations. Also, the classical limit of $N_{\mathrm{D}} \rightarrow \infty$, where the validity of the mean field theory is not in question, can easily be checked and recovered.

The structure of the present paper is as follows: we first define the screening potential and then discuss how to calculate the screening assuming the classical mean field and the dynamic method. The differences are further exposed. We then define a special interaction between particles and calculate the screening using the two methods. The results are discussed as a function of the interaction range. Conclusions and implications end the paper.

\section{The definition of the screening potential energy and factor}

Let $V_{b, 12}\left(r_{12}\right)$ be the binary interaction potential as a function of the radial distance $r_{12}$ between the particles, namely the potential between any two particles 1 and 2 in vacuum. When the two particles are part of an $N$-particles system, all particles may interact with all others and when the two particles are separated a distance $r_{12}$ the effective potential felt by each particle differs from $V_{\mathrm{b}, 12}\left(r_{12}\right)$.
The total potential energy of the system particles is given by

$E_{\mathrm{pot}}^{i}=\sum_{1 \leq i<j \leq N} V_{\mathrm{b}, i j}$

The summation is carried out over all particles. Due to the effect of the surrounding particles, the interaction energy between any two particles is written as:

$H_{\mathrm{int}, 12}\left(r_{12}, t\right)=V_{\mathrm{b}, 12}\left(r_{12}\right)+V_{\mathrm{s}}\left(r_{3}(t) \cdots r_{N}(t)\right)$

$H_{\text {int }}$ is called the effective interaction between two particles in the system. The screening potential for particles with binary potential $V_{\mathrm{b}, 12}$ is defined as:

$V_{\mathrm{s}, 12}\left(r_{12}, t\right)=H_{\mathrm{int}, 12}\left(r_{12}, t\right)-V_{\mathrm{b}, 12}\left(r_{12}\right)$.

The basic question now is how to calculate $V_{\mathrm{s}, 12}\left(r_{12}, t\right)$.

The nuclear rate enhancement is then given by $\exp \left(-V_{\mathrm{s}} / k T\right)$. The effective rate of the nuclear reaction in the system in then given to a good approximation by (Clayton p. 295):

$R=\mathrm{e}^{-V_{\mathrm{s}} / k T} n_{1} n_{2} \int_{0}^{\infty} f_{\text {rel }}\left(E_{\text {rel }}\right) \sigma\left(E_{\text {rel }}\right) v_{12} \mathrm{~d}^{3} v_{12}$

where $E_{\text {rel }}$ is the relative kinetic enerrgy at infinity, $v_{12}$ is the relative velocity and $f_{\text {rel }}$ is the distribution function of the relative kinetic energy. The screening potential is taken at the origin in the weak screening limit (which is the present case) since (a) it changes slowly as a function of space at this point, and (b) the classical turning point is much smaller than the Debye radius.

\section{Calculating the screening potential according to the mean field theory}

Mean field theory requires averaging over the potential felt by the interacting particles and tacitly assumes that the mean field is created by many particles so that the charge distribution can be considered as continuous. Hence we write:

$$
\begin{aligned}
V_{\mathrm{s}, 12}\left(r_{12}, t\right) & =\left\langle H_{\mathrm{int}, 12}\left(r_{12}, t\right)\right\rangle-V_{b, 12}\left(r_{12}\right) \\
& =\left\langle V_{\mathrm{s}}\left(r_{3}(t), \ldots . r_{N}(t)\right)\right\rangle,
\end{aligned}
$$

where the brackets \langle\rangle mean an ensemble average. The frequently made tacit assumption is that $H_{\mathrm{int}}$ is formed by many particles and fluctuations are negligible, so that the ensemble average values can be applied to the screening potential. In this limit $\left(N_{\mathrm{D}} \rightarrow \infty\right)$, the time dependence drops out, namely:

$\left\langle H_{\text {int }}\left(r_{12}, t\right)\right\rangle \rightarrow\left\langle H_{\text {int }}\left(r_{12}\right)\right\rangle$

and

$\left\langle V_{\mathrm{s}}\left(r_{12}, t\right)\right\rangle \rightarrow\left\langle V_{\mathrm{s}}\left(r_{12}\right)\right\rangle$.

In the limit of $N_{\mathrm{D}} \rightarrow \infty$ (Ichimaru 1994) the radial distribution function $g\left(r_{12}\right)$ is given by:

$\left\langle H_{\text {int }}\left(r_{12}\right)\right\rangle=V_{\mathrm{b}, 12}\left(r_{12}\right)+k T \ln g\left(r_{12}\right)$,

and the fluctuations decay to zero. This thermodynamic limit is at the base of the Alastuey \& Jancovichi (1978) result. 
The screening potential as given by Eq. (5) is supposed to be the mean potential felt by the colliding particles and expression (4) is valid when the fluctuations are negligible. As stated by Ichimaru (ibid) this potential has no dynamic role. However, the actual enhancement is $\exp \left(-V_{\mathrm{s}} / k T\right)$ and when there are fluctuations one has to take the mean $\left\langle\exp \left(-V_{\mathrm{s}} / k T\right)\right\rangle$. In the weak screening limit, when $V_{\mathrm{s}} / k T \ll 1$ the difference is expected to be negligible. However, if the fluctuations are large, the non linearity of the exponential comes into play and the effect may be large.

The following questions arise at this point:

- To what extent is $\left\langle\exp \left(-V_{\mathrm{s}} / k T\right)\right\rangle=\exp \left(-\left\langle V_{\mathrm{s}}\right\rangle / k T\right)$ ? One expects that if the fluctuations are very large there will be a strong inequality.

- The fluctuations depend on the number of particles in the interaction zone. As the potential range decreases so does the number of particles, so that the fluctuations increase, but at the same time $\left\langle V_{\mathrm{s}}\right\rangle$ decreases. How does the opposing changes in these quantities affect the final result? What happens in both limits?

- How does the result depend on the strength of the potential. Clearly, a stronger force on a short scale increases the value of the fluctuations and the potential.

\section{The dynamic method to calculate the screening}

The Molecular Dynamic method allowed us to define the screening energy from first principles in a simple and clear way.

Consider two particles 1 and 2 scattering off each other inside the $N$-body system. Let $E_{12}^{\text {rel-kin-CM, } f}$ be the relative kinetic energy when the pair is far apart and let $E_{12}^{\text {rel-kin-CM,c }}$ be the relative kinetic energy when the pair reaches the distance of closest approach. The screening energy is then given by:

$V_{\mathrm{s}}=E_{12}^{\mathrm{rel}-\mathrm{kin}-\mathrm{CM}, c}-E_{12}^{\mathrm{rel}-\mathrm{kin}-\mathrm{CM}, f}-V_{b, 12}\left(r_{\mathrm{tp}}\right)$,

where $V_{\mathrm{b}, 12}\left(r_{\mathrm{tp}}\right)$ is the binary potential interaction at the moment of closest approach. Here all energies are evaluated in the Center of Mass system. The screening energy is the energy gained/lost (from or to the particles around) by the scattered protons as they move from far away to the distance of closest approach. The potential energy of each of the the particles due to the interaction between each one and the rest of the particles is ignored when the particles are far away. This definition is very close to the original definition given by Salpeter (1954) to the screening energy (cf. Salpeter 1954, Eq. (2), where the expression for the energy is given essentially in the center of mass system). It is equally simple to define the screening energy in the laboratory system. When there are no particles with in the interaction region then $V_{\mathrm{s}}=0$, a situation that occurs for $n \rightarrow 0$ at constant $T$.

To obtain the final rate of a reaction, one has to count the number of pairs with a given $E^{\text {rel-kin-CM, } f}$ in the first case, while in the second case one has to take into account the distribution of the potential energies.

\section{The basic difference between the two types of methods to evaluate the screening}

The standard definition of the screening applies ensemble averages of the interaction and its potential between particles. The effective potential used between any two particles is obtained by continuously averaging over all particles.

The present definition is dynamic, namely the potential energy is calculated only when the two scattering particles are at the distance of closest approach so that they have converted their kinetic energy into potential one and the radial velocity vanishes.

It is expected that the two methods will converge to the same value for $N_{\mathrm{D}} \rightarrow \infty$ and will deviate from one another when $N_{\mathrm{D}} \rightarrow 0$.

\section{The short range repulsive potential}

The basic idea of the short range repulsive potential is to be able to control or parametrize the number of particles in the screening/neutralizing cloud. Also, the finite interaction range releases us from the complications of the infinite Coulomb force and the need to neutralize the positive ions, hence the calculation includes only one kind of particles which mass $m_{\mathrm{p}}$, equal to the mass of the proton.

It is well known that the effective Coulomb interaction in plasmas is short range. However, this phenomenon is not to be confused with the present approach to demonstrate the effect of the number of particles on the screening and how the statistical limit is reached. In the case of the Coulomb force, it is the play between the positive and negative charges that creates the effective short range. Here we do not discuss this effect but assume that we have imaginative particles with hypothesized short range potential.

We assume a single component system. Next, we assume the effective binary potential to be given by:

$V_{\mathrm{b}}(r)=C_{f}\left\{\begin{array}{ccc}1 / r+r / R_{n}^{2}-2 / R_{n} & \text { for } & r<R_{n} \\ 0 & \text { for } & r \geq R_{n}\end{array}\right.$

and the force is given by:

$f_{\mathrm{b}}(r)=C_{f}\left\{\begin{array}{ccc}1 / r^{2}-1 / R_{n}^{2} & \text { for } & r<R_{n} \\ 0 & \text { for } & r \geq R_{n}\end{array}\right.$

where $R_{n}$ and $C_{f}$ are free parameters. Clearly, when $R_{n} \rightarrow 0$ we get a short range force and in principle, no screening is expected. The Coulomb force is recovered when $R_{n} \rightarrow \infty$ and $C_{f} \rightarrow(1 / 2) \Gamma$, where $\Gamma$ is the Coulomb parameter and there are positively and negatively charged particles. The force and the potential are singular at the origin like the Coulomb field is. The units of the calculations are: length $\langle r\rangle=1 / n^{3}$ where $n$ is the number density. The time unit is the time needed for a particle with kinetic energy $k T$ to cross a distance $\langle r\rangle$. The unit of mass is the proton mass.

\section{The method of collecting the dynamic data}

For each particle the program searches for the nearest particle which moves towards it. There may be other particles in 
the vicinity and even closer to the particle examined. However, only the particle moving towards the selected particle is chosen. Once the close particle is identified, the motion of the pair is followed through the approach to the distance of closest approach and separation. The complete dynamic properties like kinetic and potential energies, the kinetic energy of the center of mass system, etc., are registered for later reduction. The pair is followed until their separation distance is larger then $R_{n}$. The dynamic quantities are registered upon separation and compared with those found when the particles were at the distance of closest approach. In this way the dynamics of the binary collision in the presence of many other particles is pursued in detailed. Questions like polarization of the surrounding by the approaching particles are automatically taken care off.

There are many methods to calculate the potential energy and force acting on the particle. However, due to the need for the problem at hand to follow the recoil and the energy exchange between the pair of scattering particles with the surrounding particles, it is required to calculate directly every binary interaction. Methods which rely on mean field, Poisson solvers and alike, are inappropriate since the detailed dynamics is wanted.

\section{Results}

We carried out a series of calculations in which the range of the force $R_{n}$, was changed. The thermodynamic conditions are $n=10^{26}$ and $T=1.5 \times 10^{7}$. Complete dynamic properties of the collisions were calculated. Here we discuss the effective potential felt by the scattering particles and the energy exchange between the center of mass energy of the two scattering particles and the background particles. The details of the relaxation process leading to the equilibrium and its dependence on $R_{n}$ will be discussed elsewhere. In all cases the initial conditions were random positions and Maxwellian velocity distributions. The results for the first 20 dynamics times were neglected. In the present case, a dynamic time is the time it takes a particle with energy $k T$ to cross the mean interparticle distance $\langle r\rangle$.

\subsection{The mean screening potential energy during the close approach}

The mean screening potential energy at the moment the particles are at the classical turning point $r_{\text {tp }}$ is given in Fig. 1. The figure shows the mean screening potential energy contribution to the total interaction potential between the two scattering particles at the classical turning point as a function of the relative kinetic energy at large separation (the relative kinetic energy in the CM system). The results are for several different ranges $R_{n}$. We see that as the interaction range increases the mean potential energy (for a given relative kinetic energy) becomes more independent of the relative kinetic energy, namely the curves become flatter. On the other hand, as $R_{n}$ becomes of the order of unity or less, we see that the potential energy felt by slow particles increases by up to a factor of two relative to the potential felt by the fast particles at $r=r_{\text {tp }}$. The low kinetic energy limit is essentially the adiabatic limit, however it is not quite equal to the ensemble mean potential energy of a particle

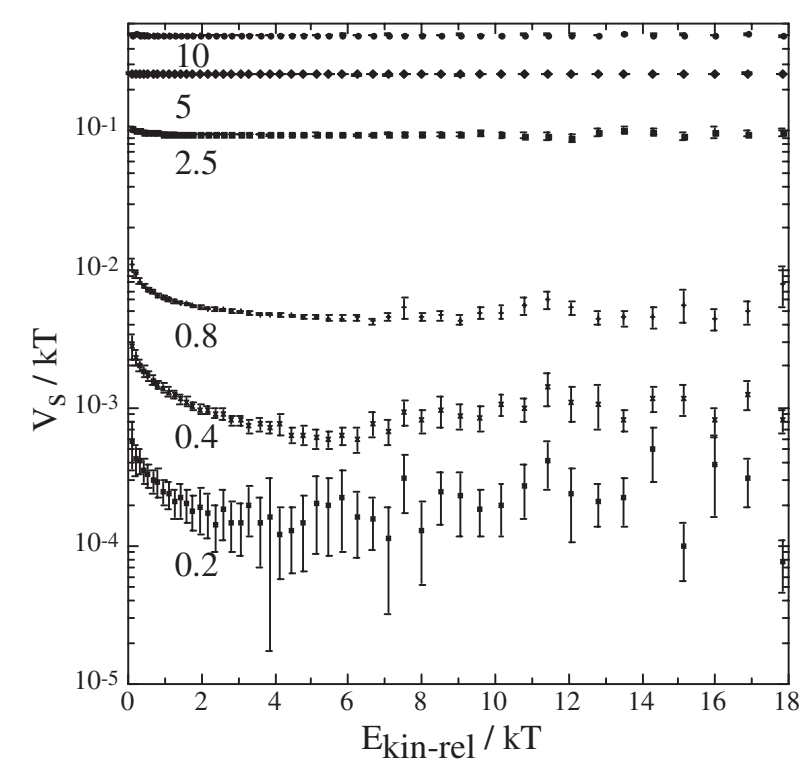

Fig. 1. The screening energy at the moment the scattering particles are at the classical turning point as a function of the relative kinetic energy at large separation. The numbers near the data refers to the corresponding range $R_{n}$.

in the system for low $R_{n}$ but approaches it as $R_{n}$ increases. This result demonstrates the expected, namely, when the scattering particles are fast, the mostly thermal particles in the surrounding respond differently from the case in which the approaching particles move very slowly and have ample time to polarize the surrounding. The numerical statistical errors shown in the figure increases with decreasing $R_{n}$ because the numerical difficulties in getting a proper statistics increase with decreasing $R_{n}$. Note, the ensemble mean potential energy of a particle, when sampled at random, is found to be independent of the kinetic energy in the laboratory exactly as predicted by Statistical Mechanics (see SS01 and SS02), here however, we ask for the potential energy at a very special moment in time and location.

In Figs. 2 and 3 we expand two cases and add to the figures an arrow with the value of the ensemble mean potential energy denoted by $\left\langle E_{\text {pot }}\right\rangle$. The latter is the classical ensemble mean potential used in the Salpeter screening theory. While for high $R_{n}$ the effect diminishes, the effect increases with decreasing $R_{n}$. The adiabatic limit obtained by moving the particles close together very slowly is reached only for extremely low relative kinetic energies. The ensemble mean potential energy per particle as a function of the interaction range is shown in Fig. 4. Also shown is the screening potential energy at the Gamow peak for the $p p$ reaction $(\approx 5 k T$ for the solar core). We find that for short effective ranges the two means differ significantly. However, as the interaction range increases well above unity, the two quantities approach each other. Thus, in the limit of a large number of particles in the neutralization zone, the two results agree.

\subsection{The distribution of the potential energy}

Of particular interest is the distribution of the potential energy among the particles. If $V_{b, 12}$ is the binary potential then the 


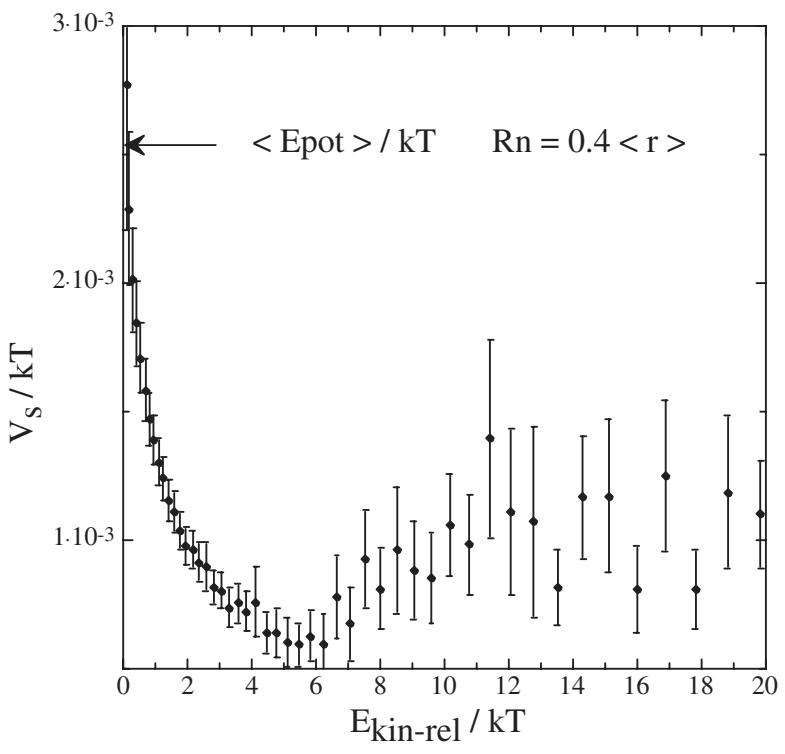

Fig. 2. The screening and ensemble mean potential energies felt by particles with given relative kinetic energy at the classical turning point. The arrow marks the ensemble mean potential felt by a particle in the system.

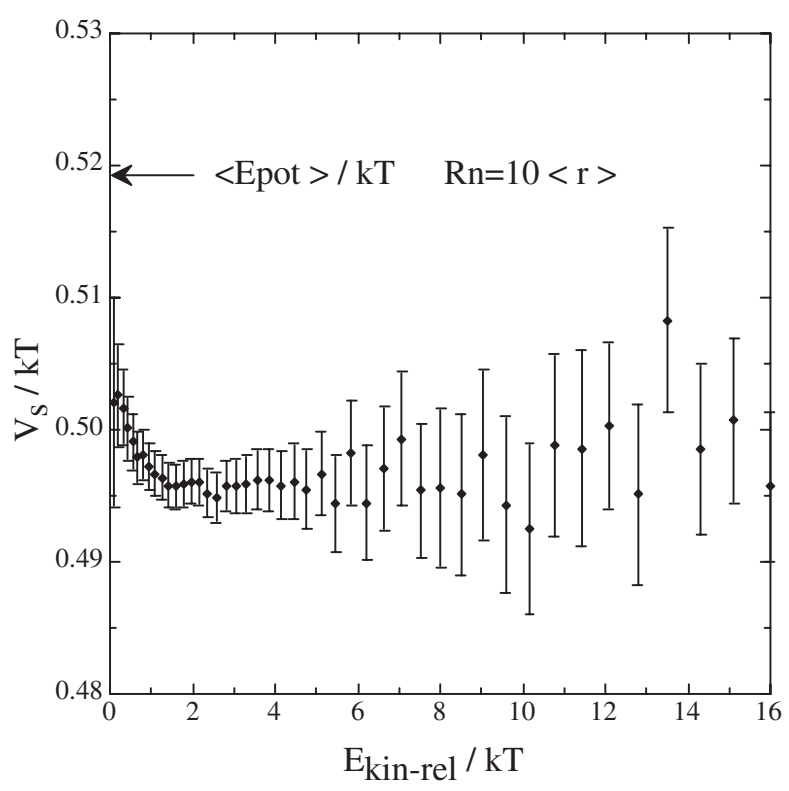

Fig. 3. The screening and ensemble mean potential energies felt by particles with given relative kinetic energy at the classical turning point for $R_{n}=10$. The arrow marks the ensemble mean potential felt by a particle in the system.

distribution should be $\exp \left(-\sum V_{b, i j} / k T\right)$, times a phase space factor, where the summation is carried over all particles. The distribution is then symmetric around the mean field value. We expect in the limit $N_{\mathrm{D}} \gg 1$ a narrow distribution the width of which is negligible for practical purposes. Indeed, we see from Fig. 5 that as the interaction range increases the distribution approaches a symmetric distribution. Also, the relative width of the distribution decreases with increasing $R_{n}$. In principle, this distribution should approach in the limit of a large number of particles in the screening radius to the one found in a snap shot.

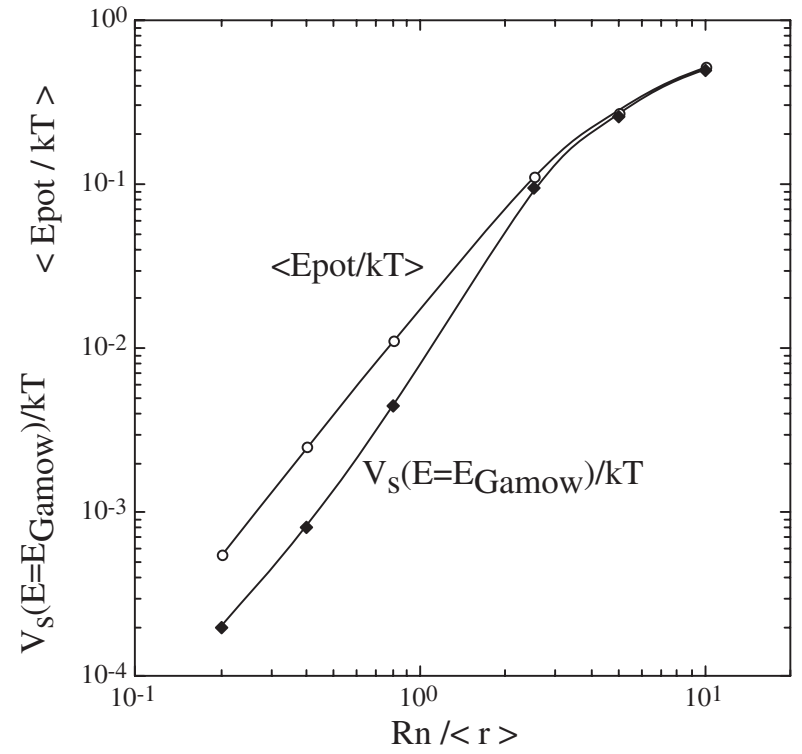

Fig. 4. The ensemble mean potential energy and the mean screening energy at the Gamow peak for the $p p$ reaction as a function of the interaction range. Here $C_{f}=1$.

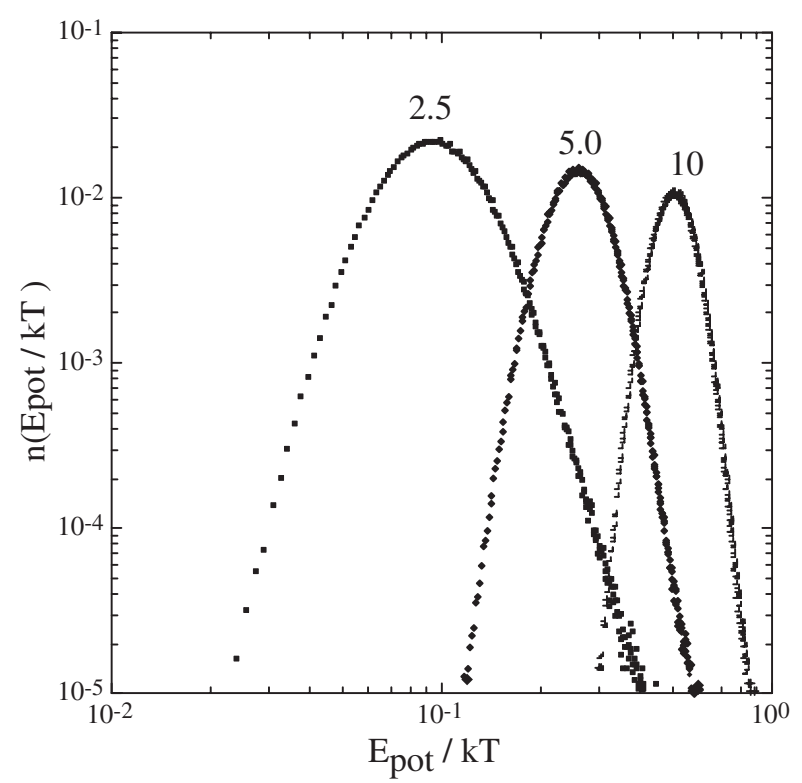

Fig. 5. The distribution of the potential energy among the particles for $R_{n}>1$. (Obtained in a snap shot calculated over all particles irrespective of their particular dynamic state.)

The situation changes when $R_{n} \leq 1$, as is seen in Fig. 6 . The striking feature is that the distribution in the latter case in not symmetric at all. Furthermore, the relative width of the distribution is very large. The deviations from a symmetric distribution is another symptom of the breakdown of the mean field assumption for the problem at hand $\left(N_{\mathrm{D}} \leq 1\right)$.

It is interesting to compare the distribution of the potential energy when the particles are far apart and when they are at the classical turning point. In Fig. 7 we show the two distributions for the case $R_{n}=5.0\langle r\rangle$. In this case the distributions were calculated over the pairs of scattering particles, not over the entire system. The distributions are marked with $f$ for far away 


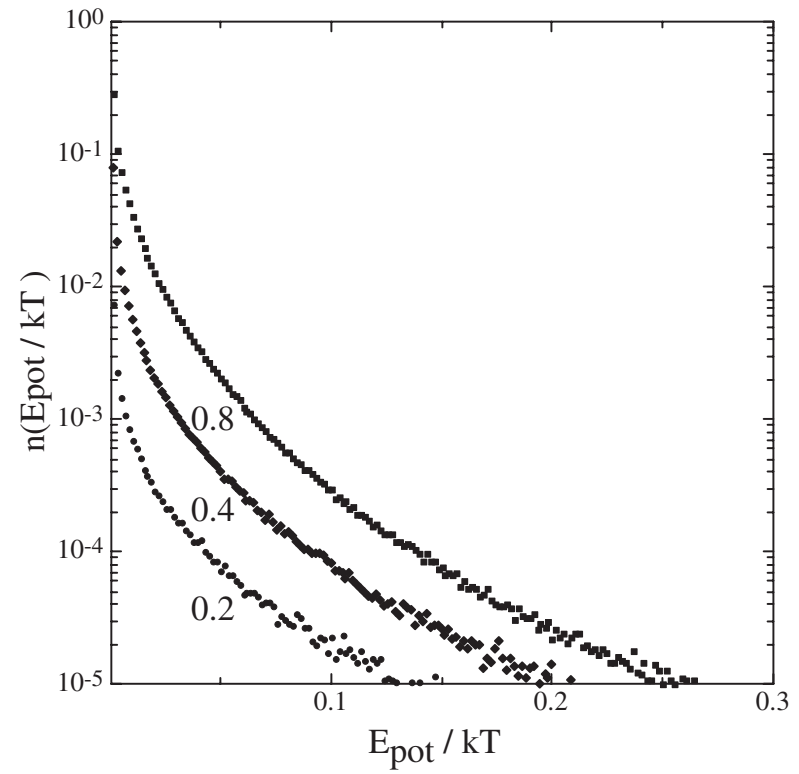

Fig. 6. The normalized distribution of the potential energy among the particles for $R_{n}<1$. (Calculated in a snap shot over all particles.)

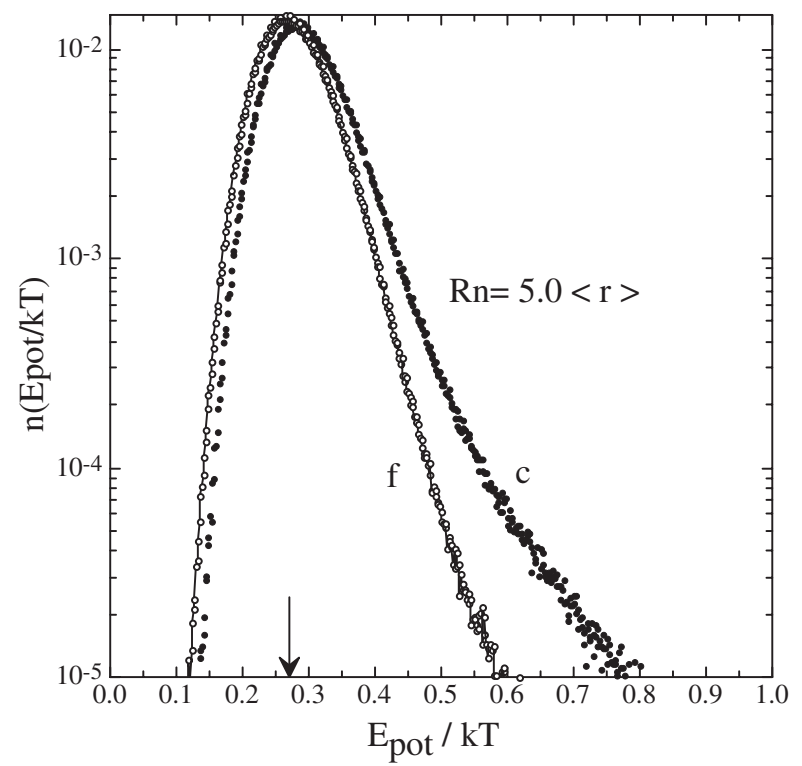

Fig. 7. The normalized distribution of the potential energy for $R_{n}=$ $5.0\langle r\rangle$ when the particles are close to each other and when they are far apart, or general particles in the system. The letters $f$ and $c$ mean $f a r$ and close respectively. The arrow points to the value of the ensemble mean.

and with $c$ for close. The $c$ distribution was registered when the two particles were at $r_{\text {tp }}$ while the $f$ distribution was registered when the same two particles separated to a distance larger than the interaction range. The arrow marks the ensemble mean and it is very close to the mean of the $f$ distribution.

The situation for the case $R_{n}=0.8<1$ is shown in Fig. 8 . The relative difference between the $f$ and the $c$ distributions is larger than in the $R_{n}=5$ case. The increase in the fluctuations as $R_{n}$ decreases is seen in Fig. 9 where the relative standard deviation $\sigma\left(E_{\text {kin-rel }}\right) / E_{\text {pot }}\left(E_{\text {kin-rel }}\right)$ is shown at each $E_{\text {kin-rel }}$ for the various ranges. The results demonstrate that for $R_{n} \leq 1$ the

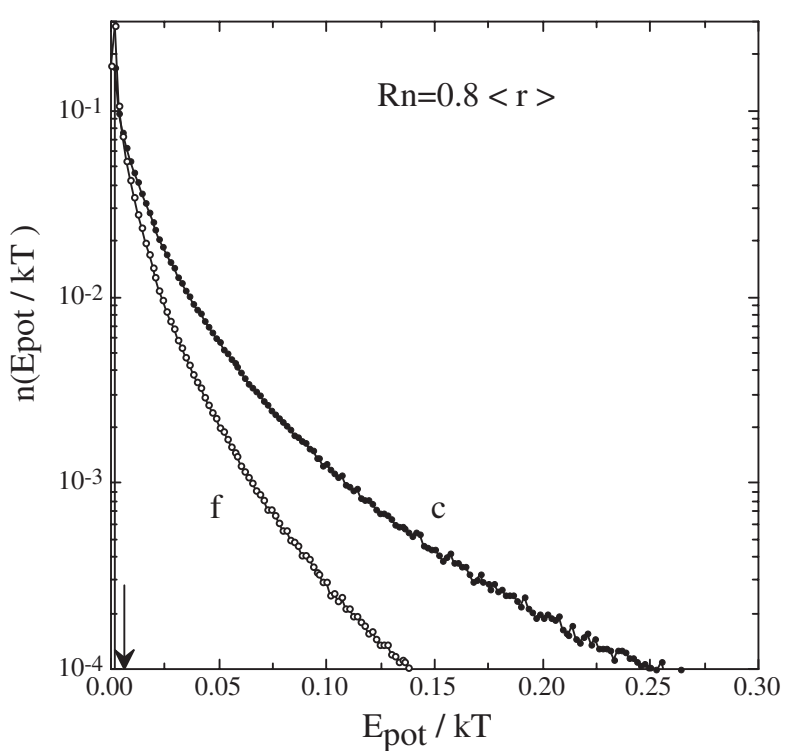

Fig. 8. The potential energy distribution for $R_{n}=0.8$. The letters $f$ and $c$ imply far and close (at the classical turning point). The arrow marks the ensemble mean.

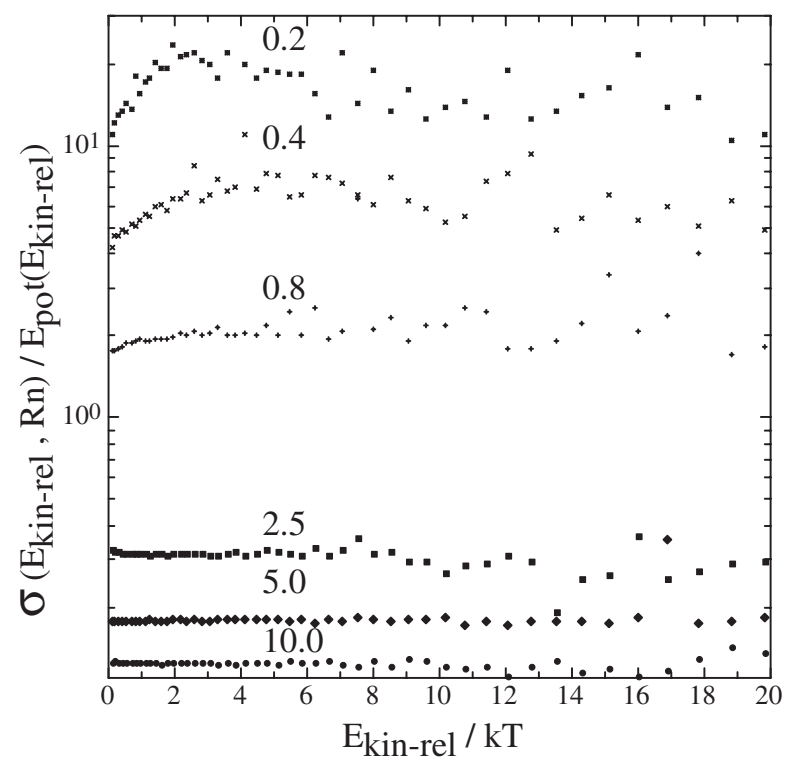

Fig. 9. The relative standard deviation as a function of the relative kinetic energy and the interaction range. The numbers are the interaction range. The figure shows how the fluctuations decay with increasing $R_{n}$.

relative fluctuations are larger than unity and $\left|\left\langle V^{2}\right\rangle-\langle V\rangle^{2}\right| \ll$ $\langle V\rangle^{2}$ does not hold.

\subsection{A stochastic potential at $N_{D} \leq 1$}

The distribution in the potential energy among particles was shown previously. Now we turn to the time dependence of the force. We expected above that the force acting on a given particle in the system fluctuates. Consequently, the potential should fluctuate as well. An example of the potential acting on a certain particle as a function of time, is shown in Fig. 10 over a single approach and separation of a pair of particle (later we 


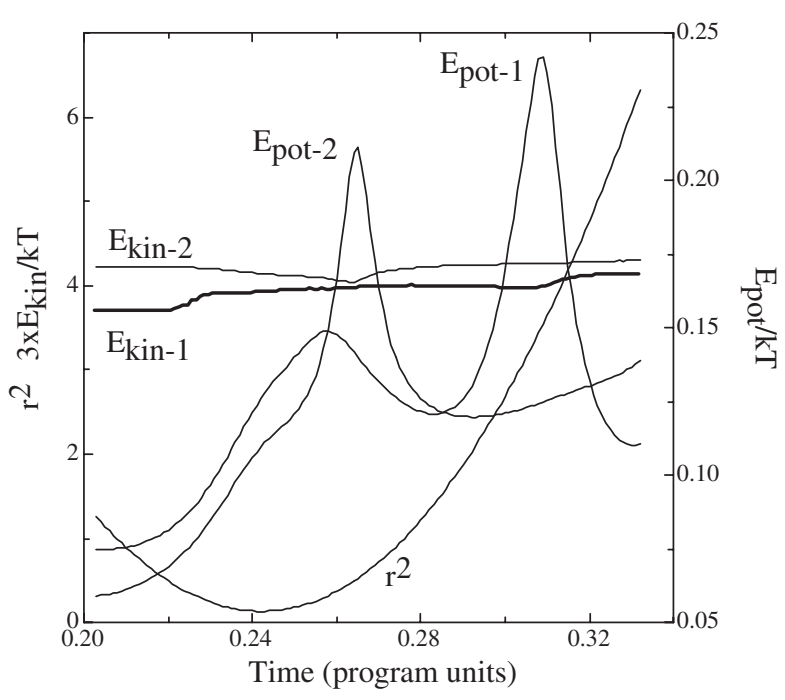

Fig. 10. The evolution of the distance squared between the approaching particles and the kinetic and potential energies as function of time for a single approach of two particles. The kinetic enegries are multiplied by 3 so as to be shown on the same scale with $r^{2}$.

show the behavour over a longer time scale). The calculation time step is $10^{-3}$ of the dynamic time (the time for a particle with energy $k T$ to cross the distance $\langle r\rangle$ ). The kinetic energies of the particles are shown as well. Particles 1 and 2 reached the distance of closest approach at $t=0.2405$ while the potential energy of particle 1 peaked at $t=0.31$ and the potential energy of particle 2 peaked at $t=0.264$. Clearly this happened due to another particle in the vicinity of particle 1 and 2 . While the kinetic energy of particle 2 reaches a local minimum at $t=0.264$, no minimum in the kinetic energy of particle 1 is seen over this span of time. We conclude that during the approach of particle 1 and 2 there were few other particles in neighborhood affecting the potential energy.

As expected, the small number of particles inside the sphere of the interaction causes temporal fluctuations in the potential felt by every particle. Note that the potential calculated is the potential felt by the particle, namely the Lagrangian potential and not the Eulerian one (the potential at a given location in space).

The emerging physical picture from the MD calculations is that of a particle interacting with a fluctuating cloud of neighboring particles (when $R_{n} \approx 1$ ). The stochastic approach can be described with a single particle Hamiltonian of the following form:

$H_{i, p}=\frac{p^{2}}{2 m}+V_{0}(r)+V_{1}(r, t)=H_{0}+V_{1}(r, t)$,

where $V_{0}$ is the static limit to the true potential. $H_{0}$ is the Hamiltonian which would lead to a mean field potential. $V_{1}$ represents the time-dependent part of the fluctuating environment around the interacting protons. A possible way to obtain the time dependent potential is:

$V_{1}(r, t)=e^{2} \int \mathrm{d} r \frac{\delta n(r, t)}{\left|r-r^{\prime}\right|}$,

where the fluctuation beyond the local two body potential is given by $\delta n(r, t)$. Once the potential is a function of time, the distribution function is not separable any longer and the effect of the screening on the scattering particles depends on the momentum of the particle. Moreover, the time dependence is required to describe the energy exchange between the variable environment and the scattered particle (see Murillo \& Weisheit 1998). We expect that $V_{1}(r, t) \rightarrow 0$ in the limit of $R_{n} \rightarrow \infty$ when the number of interacting particles tends to $\infty$.

A similar, though not identical, situation exists in the quantum domain. Alastuey (1999) analyzed the breakdown of Debye screening in quantum Coulomb systems. He finds that since in the quantum domain $\exp (-(K+V) / k T)$ cannot be factored to $\exp (-K / k T)$ and $\exp (-V / k T)$, where $K$ and $V$ are the kinetic and potential energies respectively, the potential contributions cannot be disentangled from the kinetic ones and hence dynamics enters the problem. This leads to the deviations from exponential Debye screening as conjectured by Brydges $\&$ Seiler (in the quantum domain). In our present classical case, the fluctuations induce the dynamics through an effective time dependent potential leading to the suppression of the screening.

The time dependency of the potential explains the shortcoming of using the pair distribution function to calculate the screening when $N_{\mathrm{D}} \leq 1$. The tacit assumption in the usage of $g(r)$ to calculate the screening is that the potential is defined as a function of $r$ only, which is not the case when fluctuations are important. One can of course find $g(r)$ by averaging over the fluctuations.

The dependence of the screening energy on the relative kinetic energy implies that the pair correlation function should be a function of the relative kinetic energy. In Fig. 11 we show the pair correlation function for two ranges of the potential and four bands of relative kinetic energy. We see that when $R_{n}=0.4\langle r\rangle$ the pair correlations depend on the relative kinetic energy $x=E_{\text {kin-rel }} / k T$ and

$g(r, 0 \leq x \leq 0.25)<g(r, 0.25 \leq x \leq 0.50)$

$<g(r, 0.50 \leq x \leq 0.75)<g(r, 0.75 \leq x \leq 1.00)$

namely, the pair correlation function depends on the relative kinetic energy of the particles. On the other hand, for $r_{n}=5\langle r\rangle$ we find that

$g(r, 0 \leq x \leq 0.25) \approx g(r, 0.25 \leq x \leq 0.50)$

$\approx g(r, 0.50 \leq x \leq 0.75) \approx g(r, 0.75 \leq x \leq 1.00)$

namely, the pair correlation function does not depend on the relative kinetic energy for a potential range of $5\langle r\rangle$ (the set of four curves essentially coincides).

\section{Non-central scattering}

The classical mean field assumes a two body collision with an effective spherically symmetric potential. Quite often one finds the use of an effective spherically symmetric potential of the form $\exp \left(-r / R_{n}\right) / r$, or similar ones, in dynamic problems. Namely, one assume the effect of the environment to be spherically symmetric. When the potential is spherically symmetric one expects no change in the (kinetic) energy of the center of mass system as a result of the scattering. However, when fluctuations play such a dominant role this is not the case. 


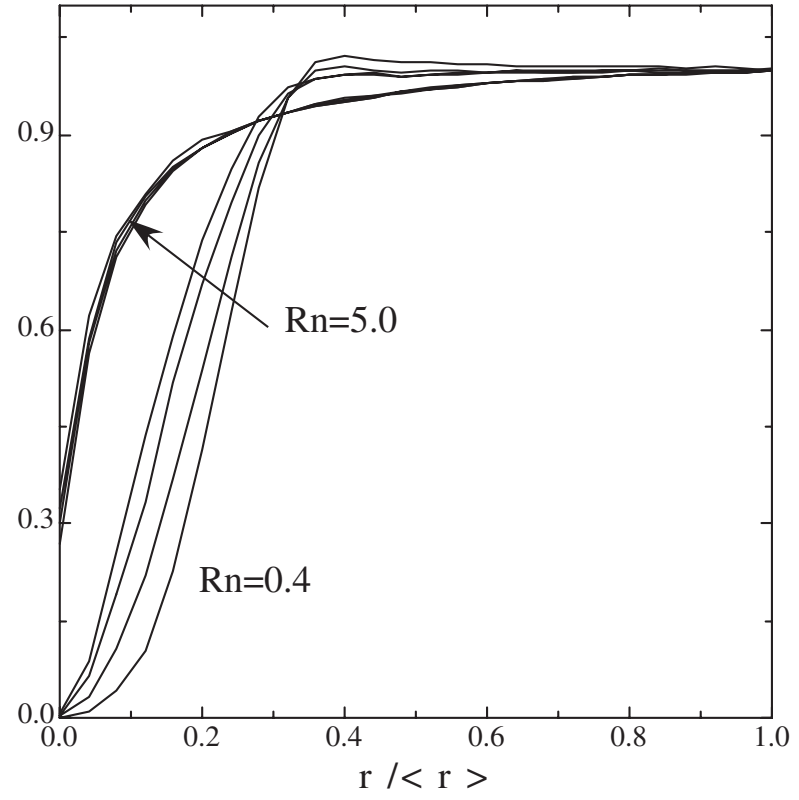

Fig. 11. The pair distribution function as a function of $r /\langle r\rangle$ and the relative kinetic energy for $R_{n}=0.4$ and 5.0. The two set of lines are the pair correlation for the following relative kinetic energy bands: $0-0.25 k T, 0.25-0.50 k T, 0.50-0.75 k T, 0.75-1.00 k T$.

Define the mean absolute change in the energy of the center of mass as $\left\langle\left|E_{\text {kin-CM }}^{f}-E_{\text {kin-CM }}^{c}\right|\right\rangle=\left\langle\left|\Delta E_{\text {kin-CM }}\right|\right\rangle$, where $E_{\text {kin-CM }}$ is the kinetic energy of the total mass between the moment of closest approach and separation (to a distance $r \geq R_{n}$ ). In other words, $E_{\mathrm{kin}-\mathrm{CM}}=m\left(v_{i}+v_{j}\right)^{2}$ for a pair $i j$ of scattering particles.

In Fig. 12 we show how $\left\langle\left|\Delta E_{\text {kin-CM }}\right|\right\rangle$ varies as a function of three possible independent variables: (a) the relative kinetic energy in the center of mass system, namely the kinetic energy of the reduced mass (cf. panel a), (b) the total kinetic energy of the particles in the laboratory at large separation, namely as a function of $E_{\text {tot-kin-far-lab }}=(1 / 2) m\left(v_{f, 1}^{2}+v_{f, 2}^{2}\right)$, and (c) as a function of kinetic energy of the center of mass system when far away, namely $E_{\text {kin-far-CM }}=m\left(v_{f, 1}+v_{f, 2}\right)^{2}$. The example is calculated for the case $R_{n}=0.8\langle r\rangle$. In all panels, + are for $\left\langle\Delta E_{\mathrm{CM}}\right\rangle$, full circles are for $\left\langle\left|\Delta E_{\mathrm{CM}}\right|\right\rangle$ and full squares are for the standard deviation $\sigma\left|\Delta E_{\mathrm{CM}}\right|$. All quantities are normalized to the ensemble average potential energy.

We remark a large dependence on $E_{\text {tot-kin-far-CM }}$ and $E_{\text {kin-far-CM }}$, which are measured in the laboratory and a weak dependence on $E_{\text {kin-rel-CM }}$ which is measured in the center of mass system. Thus, the motion of the particles relative to the laboratory affects the change in the energy of the system of mass. The standard deviation is large.

We observe that the result for the mean value of $\left|\Delta E_{\mathrm{CM}} / k T\right|$ as a function of $E_{\text {kin-CM-far }}$ is significantly lower than the corresponding change as a function of the relative kinetic energy. (Note the mean is carried out over all cases in the problem). Moreover, when the number of cases considered increases and with it the statistics, the lower curve $\left(\Delta E_{\mathrm{CM}}\right.$ with respect to $E_{\text {kin-CM-far }}$ goes down, unlike the curve with respect to the relative kinetic energy. The implication is that the change

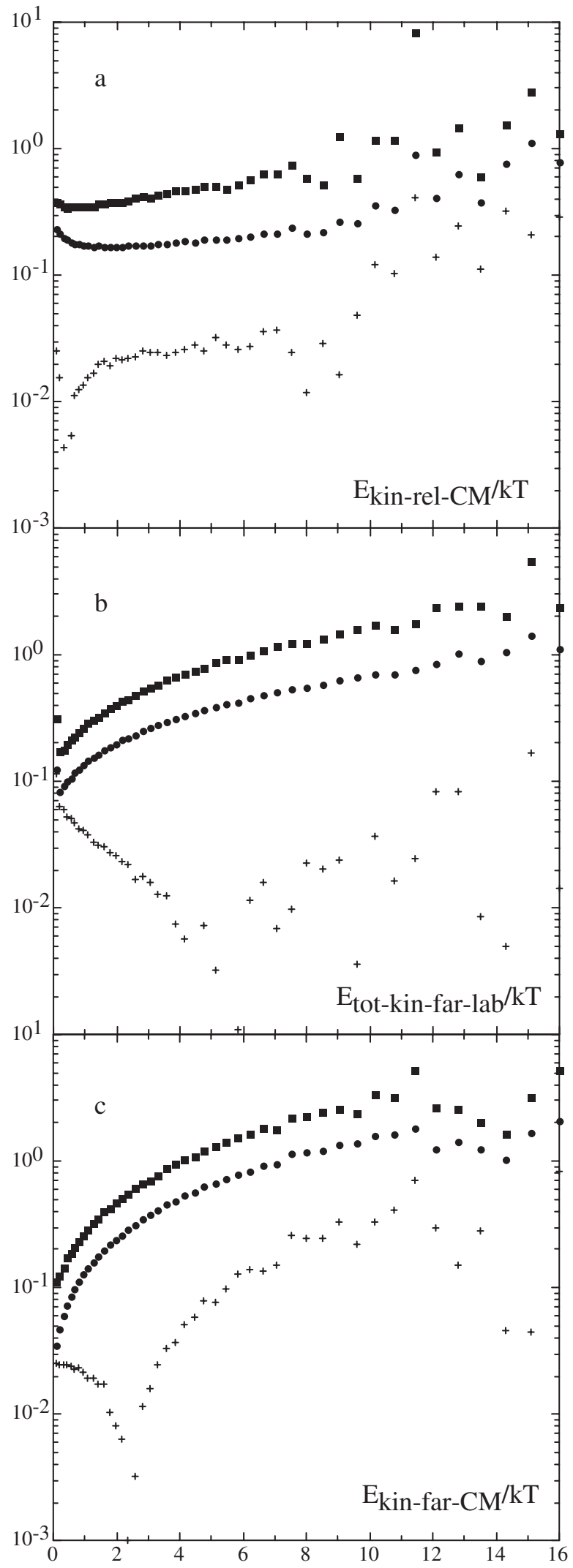

Fig. 12. The distribution of $\left\langle\Delta E_{\text {kin-CM }}\right\rangle /\left\langle E_{\mathrm{pot}}\right\rangle$, marked with + signs, $\left\langle\left|\Delta E_{\text {kin-CM }}\right|\right\rangle /\left\langle E_{\text {pot }}\right\rangle$, marked with full circles and $\sigma\left|\Delta E_{\text {kin-CM }}\right| /\left\langle E_{\text {pot }}\right\rangle$, marked with full squares, as a function of kinetic energy of the reduced mass in the center of mass system a), as a function of the total kinetic energy of the pair in the laboratory system b) and total kinetic energy of the center of mass system when far apart c). 


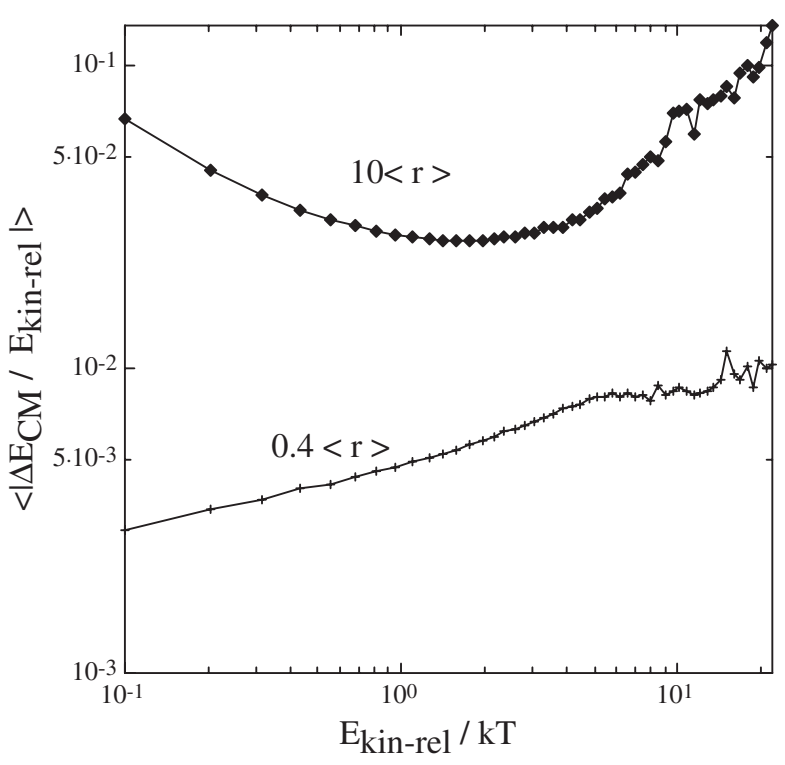

Fig. 13. The mean change in the of the center of mass as a function of the relative kinetic energy at large separation. Shown are the results for $R_{n}=10\langle r\rangle$ and $R_{n}=0.4\langle r\rangle$.

in the center of mass energy does not depend on the energy of the $\mathrm{CM}$ system relative to the laboratory and depends primarily of the relative kinetic energy in the CM system.

The importance of the absolute energy change of the CM system can be assessed from Fig. 12 and the relative change can be seen in Fig. 13 where just two cases are shown. In view of the role of fluctuations one would have expected that the change in the CM energy would decrease with $R_{n}$ but the inverse is found. The plausible explanation is that the change depends on the extent of the deviation of the collision from a pure two body collision. When the range is small, there are very few extra particles within the interaction range to have an effect. On the other hand, as the range increases, so does the number of particles. However, a $R_{n}$ of $10\langle r\rangle$ is not large enough to contain a sufficiently large number of particles to average the change to zero.

The fact that the scattering takes place via a non central force and energy is gained/lost by the CM system results in the consequence that not all the available potential energy goes to affect the radial motion. Part of it is taken by the CM system and is not available for affecting the screening and the rate of the reaction. In the figure the energy change is shown as a ratio of the kinetic energy. However, one should consider the effect also relative to the corresponding screening energies, $5 \times 10^{-3} k T$ and $0.4 k T$ respectively.

\section{The dependence on the force constant}

It is of interest to see the dependence of the quantities under consideration on the force constant. So we increased the force constant artificially. Clearly, when related to stellar plasma the increased force constant relates to particle with a higher charge (but the same mass). Figure 14 provides a comparison of $V_{\mathrm{s}}$ between the cases $C_{f}=1$ and $C_{f}=10$ for the same range of the force. The statistics for $C_{f}=10$ and $E_{\text {kin-rel }}>10 k T$

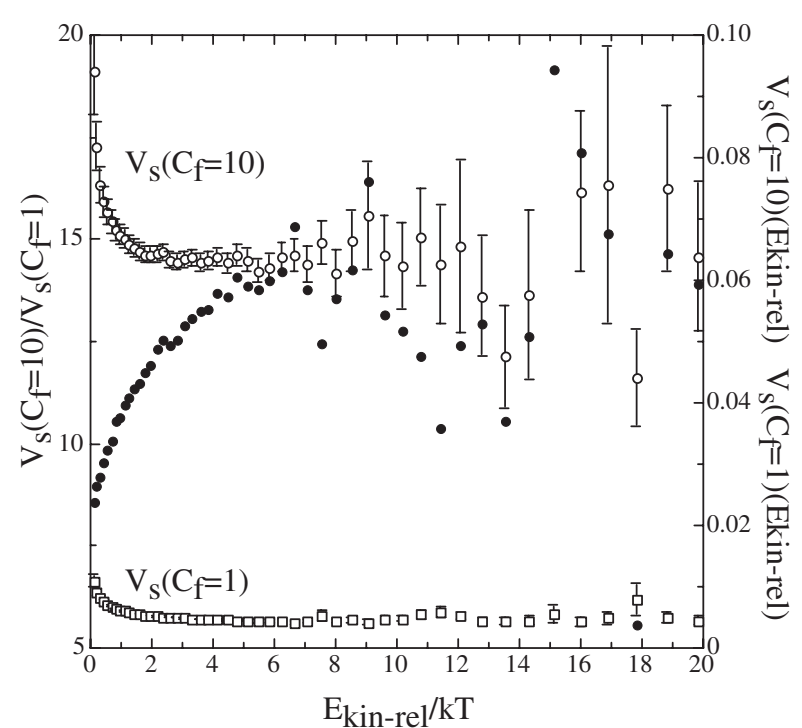

Fig. 14. The effect of the force constant, Shown are the screening energy $V_{\mathrm{s}}$ at the classical turning point for two cases: $C_{f}=1$ (open squares) and $C_{f}=10$ (open circles) as a function of the relative kinetic energy. The ratio between the two as a function of relative kinetic energy is showen as well (as filled circles)

is not good leading to large statistical errors. Longer calculations show that at $\mathrm{d} V_{\mathrm{s}} / \mathrm{d} E_{\text {kin-rel }}$ decreases very quickly for low energies and much slowlier at high energies and the change is monotonic. While the general behavior is the same the increase in the force constant does not increase the screening energy equally at all relative kinetic energies and hence simple scaling is precluded. However, we find that $\left\langle V_{\mathrm{s}}\left(C_{f}=10\right)\right\rangle /\left\langle V_{\mathrm{s}}\left(C_{f}=\right.\right.$ $1)\rangle=9.95 \pm 0.06$

\section{The screening as a function of $R_{n}$}

The screening correction for the tunnneling through the potential was calculated as a function of the relative kinetic energy at large separation and compared with the classical expression calculated from the mean potential energy per particle in the system. Let the rate of the reaction in infinite dilution be given by $R_{0}$ and the acceleration factor by the screening at a finite density by $f$ so that the rate at a finite density is $R=f R_{0}$. We define the enhancement $h$ by $h=f-1$. In Fig. 15 we give the ratio of the new value of $h$ (as found here) to $h_{\text {sal }}$, the old Salpeter value for two values of the potential strength $\left(C_{f}=1\right.$ and $\left.C_{f}=10\right)$. In the case of the old Salpeter value we have $h_{\text {sal }}=\exp \left(E_{\text {Debye }}\right)$, where $E_{\text {Debye }}$ is the Debye potential energy per ion. We see that as the range increases the ratio approaches unity from below. Namely, (a) the effect of the fluctuations decreases with increasing $R_{n}$ and (b) the fluctuations usually reduce the screening. On the other hand, as the range decreases to below unity marked deviations take place. The positive contributions of the fluctuations are unable to overcome the negative ones. The mean potential energy decreases but the effect of the fluctuations decreases slowlier. Note that the distribution of the potential energies changes as well. The arrow marks the value of the Debye radius in the case of the pure Hydrogen plasma at $n=10^{26}$ and $T=1.5 \times 10^{7}$. Thus the effect of screening in the 


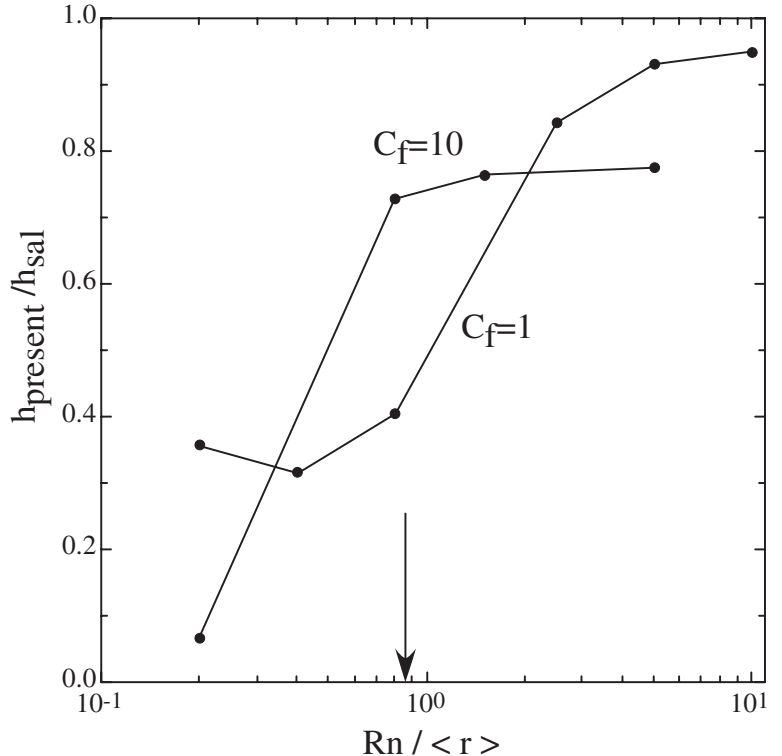

Fig. 15. The ratio of the new screening enhancement to the old screening enhancement factor as a function of the range of the potential for two values of the potential strength.

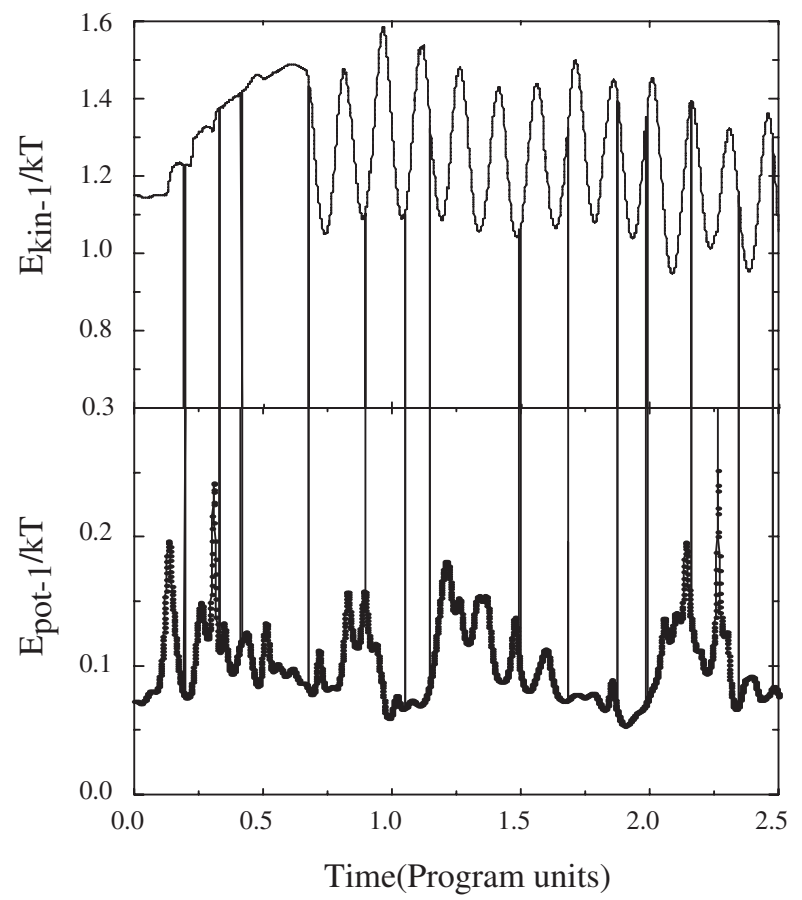

Fig. 16. The time dependence of the kinetic and potential energy of a given fixed particle. Vertical straight lines indicate when the pair is broken and a new "close particle" is selected.

Sun a là Salpeter classical theory is over estimated by a factor of 2.5. (Detailed enhancement factors for the Sun will be given in a separate communication).

\section{The effective screening}

As the potential is time dependent the tunneling through the potential becomes the problem of tunneling through time dependent potential. It is therefore important to compare the time

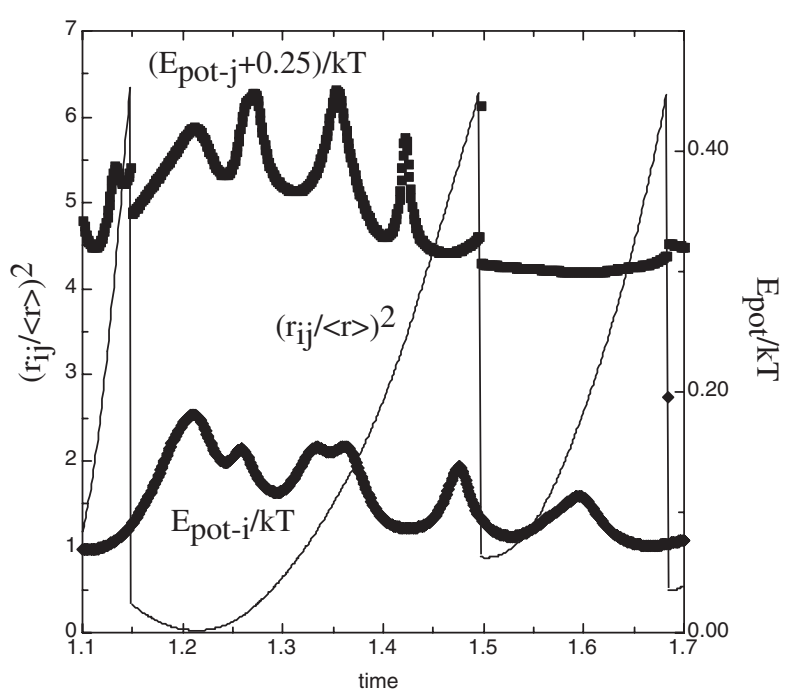

Fig. 17. The details over a short time span of the potential energy of the particle (marked by $E_{\mathrm{pot}}(i)$, the potential energy of the second particle (marked by $\left.E_{\text {pot }}(j)\right)$ and the square of the distance between particle 1 and $j$ (marked with $r_{1 j}^{2}$ ).

scale for the major fluctuations with the tunneling time scale. In Fig. 16 we gain some insight a typical time evolution of the particle's kinetic and potential energy. The time units is the time it takes a particle with the mean kinetic energy to cross the mean inter particle distance. In this case $R_{n}=2.5\langle r\rangle$. The upper panel shows the kinetic energy while the lower panel shows the potential energy. The vertical lines are the moment the pairing changes, namely a new close particle is found (after the previous one distanced beyond $R_{n}$ ). First, the kinetic energy increases gradually and then oscillates as the particle scatters off different other particles. It so happened that the particular particle chosen for the follow up is first accelerated by the collision with few particles and only then starts a sequence of oscillations with a gradual decrease in kinetic energy. Second, the potential energy fluctuates and reaches even relatively very high peaks. However, it is important to see what happens on a much shorter time scale.

For that we show in Fig. 17 the time evolution over an expanded time axis. The rather fast fluctuations over a long time scale appear as slow variations in the potential energy. In particular, in the vicinity of the minimum in $r_{1 j}^{2}$, the potential is slowly varying. The vertical lines in the curve of $r_{i j}^{2}$ indicate the moments at which the new close particle is chosen and $r_{1 j}^{2}$ jumps from the particle that distanced away to a new close particle. We also note that the potential energy of the particle traced, particle $i$, is continuous. On the other hand, the potential energy of the close particle changes abruptly as the neighboring particle changes. Thus the curve $E_{\mathrm{pot}-j}$ refers to the potential energy of three different particles at three different time sections. The conclusions drawn from the particular case shown here are corroborated by considering the relation between the amplitude of the fluctuation as the frequency. The very fast fluctuations have a very small amplitude (cf. SS01). Thus the adiabatic limit in which the external fluctuations are 
frozen during the tunneling is plausible. All the calculations presented here assume this assumption.

\subsection{The approximation of constant potential during tunneling}

The tunneling time through the potential barrier can be derived by substituting (Kleber 1994) $t=i \tau$ and solving the energy conservation equation for the trajectory, namely:

$\left(\frac{\mathrm{d} r}{\mathrm{~d} \tau}\right)^{2}=\frac{2}{m}\left[V_{b, 12}(r)+V_{\mathrm{s}}(r, t)-E_{\mathrm{kin}-\mathrm{rel}, f}\right]$.

The solution for the tunneling time as a function of the kinetic energy is

$$
\begin{aligned}
\tau_{\text {tun }} & =\sqrt{\frac{2}{m}} \int_{r_{\text {tp }}}^{0}\left(V_{b, 12}(r)+V_{\mathrm{s}}(r)-E_{\mathrm{kin}-\mathrm{rel}, f}\right)^{-1 / 2} \mathrm{~d} r \\
& =O(1) \frac{C_{f}}{E_{\mathrm{kin}-\mathrm{rel}, f}^{3 / 2}} .
\end{aligned}
$$

Clearly, the approximation breaks down for $E_{\mathrm{kin}-\mathrm{rel}, f} \rightarrow 0$, but this limit is not important for the reaction rate. Actually, the above estimate does not take into account the fact that the relative magnitude of the fluctuation becomes negligible after the particle penetrates a certain distance and the binary potential dominates the tunneling. Hence, it is safe to assume in the weak screening approximation that the fluctuation remains constant during the tunneling and the effect of the fluctuation can be estimated by assuming a constant in time potential.

\section{Conclusions}

We have carried out a numerical experiment in which the finite range of the interaction and its strength are varied. The variation went from the region of short range, even smaller than the mean inter particle distance, to a high range of up to 10 mean inter particle distances. Thus the behavior of the system could be followed as the number of particles in the neutralizing domain is changed from very few to many. The numerical experiment allows us to single out the peculiarities of systems with a short range (effective) potential and obtain the limit of a large number of particles in the neutralization domain. In particular, we find that

- The screening deviates significantly from the classical value as soon as the neutralization radius approaches unity from above (the density increases at a constant temperature).
- The standard mean field results for the screening deviate from the values obtained for a small numbers of particles. Assuming the mean field approximation a 1 à BBGS leads to errors in the estimates of the screening enhancement.

- The effects are not linear in the strength of interaction and vary with relative kinetic energy. The ensemble averaged potential, though varies linearly with $C_{f}$.

- The screening potential energy $V_{\mathrm{s}}$ must be derived from dynamic considerations when $R_{n}=O(1)$. As the particles approach each other their potential polarization effect cause a deviation of the screening energy from the ensemble averaged potential energy.

- The present results are a numerical experiment in the behavior of an hypothetical gas. However, a plasma composed of positive and negative charges possess an affective neutralizing radius - the Debye radius. We expect that a plasma will show the same effects when the Debye radius replaces $R_{n}$.

Results for the screening in a real plasma will be published in a forthcoming paper.

Acknowledgements. The author is happy to acknowledge a discussion with M. Fisher about fluctuations. Extensive discussions with N. J. Shaviv are acknowledged. This research was partly supported by the Foundation for the Promotion of Research at the Technion and by the Asher Foundation at the Asher Space Research Institute. Partial support by the DFG (Sonderforschungsberiech 359/C2) is acknowledged.

\section{References}

Alastuey, A. 1999, Physics A, 263, 271

Alastuey, A., \& Jancovici, B. 1978, ApJ, 226, 1034

Bahcall, N. J., Brown, L. S., Gruzinov, A., \& Sawyer, R. F. 2001, A\&A, 383, 291 (BBGS)

Brydges, D. C., \& Seiler, E. 1988, J. Stat. Phys., 42, 405

Clayton, D. D. 1968, Principles of Stellar Evolution and Nucleosynthesis (McGraw-Hill)

Ichimaru, S. 1994, Statistical Plasma Physics (Addison-Wesley pub), 37

Landau, L. D., \& Lifshitz, E. M. 1959, Statistical Mechanics (Pergamon Press), 345

Murillo, M. S., \& Weisheit, J. C. 1998, Phys. Rep., 302, 1

Salpeter, E. E. 1954, Aust. J. Phys., 7, 373

Salpeter, E. E., \& Van Horn, H. M. 1969, ApJ, 155, 183

Schatzman, E. 1948, J. Phys. Rad., 9, 46

Shaviv, G., \& Shaviv, N. J. 2000, ApJ, 529, 1054 (SS00)

Shaviv, G., \& Shaviv, N. J. 2001, ApJ, 558, 925 (SS01) 